\title{
A Qualitative Study of Home Care Client and Caregiver Experiences With a Complex Cardio- respiratory Management Model
}

Connie Schumacher ( $\nabla$ cschumacher@brocku.ca )

Brock University

Darly Dash

McMaster University

Fabrice Mowbray

McMaster University

Lindsay Klea

McMaster University

Andrew Costa

McMaster University

\section{Research Article}

Keywords: Home care, nursing, self-care, self-management, emergency avoidance, model of care

Posted Date: January 19th, 2021

DOl: https://doi.org/10.21203/rs.3.rs-145167/v1

License: (c) (i) This work is licensed under a Creative Commons Attribution 4.0 International License. Read Full License 


\section{Abstract}

\section{Background}

Home care clients are typically older and have some degree of medical, physical, cognitive or social conditions that require formal or informal support to promote healthy aging in the community. Home care clients contribute a significant proportion of health service use, including emergency department visits. The DIVERT-CARE trial introduced a cardio-respiratory management model to improve patient motivation, symptoms and rates of unwarranted health service use. Our objective was to explore the perceptions and experiences of individuals who participated in the DIVERT-CARE self-management support and education intervention.

\section{Methods}

A qualitative study was nested within a pragmatic randomized control trial and conducted following a 15week multicomponent cardio-respiratory intervention. A phenomenological descriptive design was employed using thematic analysis. Post-intervention, clients and their caregivers were invited to participate in a semi-structured telephone interview. Interview questions were designed to elicit the experience with the intervention components.

\section{Results}

A total of 29 interviews were completed from June 2018 to March 2020 from participants in Ontario, Newfoundland, and British Columbia. Five themes were identified; self-care trajectory and responsibility, learning and behaviour change, pre-emptive connection to care providers, connection to information and the health system, and connection through therapeutic relationships.

\section{Conclusions}

Home care clients experience unique challenges in managing chronic disease. Home-based interventions fostered a therapeutic relationship of connectedness while equipping clients with necessary knowledge and skills. These results inform recommendations for community nursing, and home-based selfmanagement supports for older community-residing individuals.

\section{Introduction}

Home care $(\mathrm{HC})$ clients are a large and expanding group of medically complex older adults with poor access to effective chronic disease management (1). Limited community-based resources and budgetary constraints leave many HC clients with unmet care needs (2). These individuals frequently turn to the emergency departments (ED) for medical care, contributing twice as many visits when compared to other older adult populations (3). Time pressures and high patient volumes hinder emergency clinicians from addressing geriatric complexity and chronic disease management in the ED $(4,5)$. Greater emphasis on 
improving community-based disease management and service integration is recommended to support the multifaceted needs of older adults and prevent unwarranted ED visitation (6).

$\mathrm{HC}$ clients with cardiopulmonary disease or symptoms (e.g., angina, dyspnea, arrhythmias, etc.) are at greater risk for clinical decompensation and frequent health service utilization. To better identify and support these individuals in the community, we are currently conducting a multicenter pragmatic clusterrandomized control trial, herein referred to as the DIVERT-CARE Trial. The objective of this trial is to evaluate the effectiveness of a cardio-respiratory disease management model for improving patient motivation, reducing symptoms, prolonging time to unplanned ED visits and health care cost(1). HC caseloads were randomized to either receive the DIVERT-CARE intervention or the standard of care. In treatment clusters, clients identified as high-risk for ED visitation were offered a bundle of interventions including scheduled nurse-led self-management support, access to a staff helpline, education on vaccines, advance care and goal planning, medication reconciliation with a pharmacist and documented recommendations to support continuity-of-care in the community $(1,7)$.

The integration of qualitative research methods in clinical trials is common, as they shed light on contextual factors surrounding trial findings, methods employed, feasibility and participant experiences $(8,9)$. The DIVERT-CARE Trial will aim to establish the effectiveness of the model. However, understanding the participant experience, preferences, and delineating which interventions are wellreceived is equally important, as $\mathrm{HC}$ clients have a primary role in their care and self-management. Our objective was to explore the perceptions and experience of $\mathrm{HC}$ clients with chronic diseases after the introduction of the DIVERT-CARE intervention.

\section{Methods}

We conducted a phenomenological descriptive study that was nested within the DIVERT-CARE trial and conducted at the end of the 15-week client intervention period. As part of the intervention, $\mathrm{HC}$ clients received congestive heart failure (CHF) or chronic obstructive lung disease (COPD) zones reference sheets (10). The zones are one-page symptom monitoring decision aids for CHF and COPD. The qualitative study was conceptualized and designed in parallel to the trial protocol (1)This study was performed in accordance with the Declaration of Helsinki and approved by the Hamilton Integrated Research Ethics Board, Island Health Research Ethics Board, and the Newfoundland and Labrador Health Research Ethics Board. This study was funded by the Canadian Institutes of Health Research (grant \# 148933).

\section{Research Question}

What are the experiences and perceptions of $\mathrm{HC}$ clients or their caregivers concerning various components of the DIVERT-CARE cardio-respiratory management model?

\section{Participants and Study Setting}


Following a 15-week community-based multicomponent cardio-respiratory intervention, $\mathrm{HC}$ clients or their caregivers were recruited to participate in a telephone-based interview. Participants were eligible for study inclusion if they participated and completed at least one component of the intervention, were English speaking and provided a contact telephone number. Where the recipient of care was not autonomous in their chronic disease management, their caregiver was approached to gain a contextual understanding of their experience. Participants who consented to further contact post-intervention were contacted by the research coordinator by telephone (DD, LK). For this purposive sample, we aimed for representation of both males and females, a sample size of 30 , and representation from all three regions participating in the DIVERT-CARE intervention. Interviews were completed from June 2018 to March 2020.

\section{Data Collection and Analysis}

A date and time were arranged to complete the interview with a research coordinator (DD, LK). Participants provided informed verbal consent that was audio-recorded prior to the interview. Semistructured interviews with clients or caregivers were conducted using an interview guide (found in Additional File 1). The interview guide was structured to elicit experiences with the DIVERT-CARE intervention components, the perceived effectiveness of the intervention components or changes to managing their health, communication with care providers, and areas for improvement to better suit their care needs. Interviews ranged from nine to 57 minutes, averaging 27 minutes. Data were transcribed verbatim and anonymized for analysis. Participants were not offered reimbursement for participating.

Qualitative data were housed in QSR International NVivo 12 Pro. We analyzed the data using a phenomenological thematic analysis technique, following these steps: familiarization with the data, generate initial codes, search for themes, review themes, define and name themes, and produce the report (11). To validate coding, each transcript was independently read and analyzed by two members of the research team (CS and LK). The process involved line-by-line coding of the first five interviews, after which the coding was compared, and the coding framework was developed. All other interviews were then coded independently, and the codebook was further refined (12). Frequent meetings were held between two researchers (CS, LK) to discuss the meaning units, alignment with the codebook, and any emerging codes. The codes were examined with a constant comparison approach by four researchers (CS, LK, DD, and FM) to discuss and refine the emerging themes. Themes were derived from the data, by focusing on patterns and descriptions of the experiences related to the research question. All data were given equal consideration during analysis, and codes were reduced into themes that were adequately descriptive. Saturation was reached during analysis where the research question was answered through these recurring themes.

Reliability and validity were checked by two researchers (CS, DD), where analysis and interpretation were continuously monitored and systematically compared back to the data(13). Representative quotes were selected from the interviews to highlight key findings. We used the Consolidated Criteria for Reporting Qualitative Research (COREQ) checklist to guide the reporting of this study(14) (Additional File 2). 
Specific strategies used to attain trustworthiness included member checking post interview, peer debriefing, audit trails, and analysis of negative cases (15).

\section{Results}

\section{Participants}

A total of 70 individuals consented to be approached for follow-up contact. Of these, 56 were contacted to request participation in the present study. We were unable to reach 10 participants and 11 refused to participate, reporting a lack of time or interest as reasons for refusal. We conducted 29 interviews with consent from six males and 23 females; 20 participants were $\mathrm{HC}$ clients and nine were caregivers. Home care clients whose perceptions are reported in this paper were aged 51 years to 98 years with a mean of 78 years. 19 interviews were from Ontario, four from Newfoundland, and six from British Columbia. Five themes emerged from the thematic analysis: self-care trajectory and responsibility, learning and behaviour change, connection to care provider, connection to information and the health system, and connection through emotional support and caring. Representative quotes are included below with pseudonyms for participants and were classified as ' $\mathrm{C}$ ' for $\mathrm{HC}$ clients and ' $\mathrm{CG}$ ' for caregivers, and as one, two or three in accordance with the study site. Supplemental quotes are provided in Table 1.

\section{Self-care Trajectory and Responsibility}

Participants described their experience with the DIVERT-CARE intervention within the context of self-care and their journey managing chronic disease symptoms and treatment. We asked how managing their care had changed following the provision of health education and support in their home. Many described this change by first elaborating on their experiences caring for themselves over the course of their disease, including perceived capabilities, feelings, and uncertainties. Several HC clients and caregivers acknowledged a lack of confidence in their ability to provide self-care.

'Well see, another bad mistake with a lot of people, and I was like, feelin' guilty of that myself, you think you know all of it. But never be afraid to find out more because, ya know, there's times over the years, not in the last couple of years, but over the years since 1996, there's things that I've been doing that I'm not doing it right. Like I thought I was doing it right' $\mathrm{Bob}(\mathrm{C}, 1)$

The daily toll and responsibility of caring for one-self and navigating changes in health were likened to feelings of burden. Common daily symptoms that were reported included shortness of breath, and edema, most often seen in the lower extremities. Some participants highlighted that shortness of breath seemed difficult to manage and lacked distinct features where actions should or could be taken. For instance, one respondent described that their bucket was full, giving a sense that they were overwhelmed with ongoing monitoring and self-care responsibilities. An inability to alleviate symptoms was common among participants and evoked feelings of fear, uncertainty, and inaction. 
'I have shortness of breath again and I have my feet are swollen. I never thought nothing of it because of the fact that I've always had this shortness of breath, right?...So, anyway, I'm diagnosed with that now, so I don't know what's- what's going to be next. My bucket is almost full.'Keith (C, 3)

'It scares the hell out of me sometimes. Yeah, I get really scared and then I just, I think - what am I going to do, like what am I going to do? And - I'm not going to treat this anymore because it is too much, and uh, like, I can't breathe [crying]... Not everything, but maybe one of the most important things, you forget. 'Oh God', you know? What am I going to do? What should I do? I forget, I can't remember.' Lori (C, 1)

Many reported concerns about the impact that their care was having on the lives of their families, and that they desired to take sole responsibility for their care. To illustrate, $\operatorname{Jim}(C, 1)$ shared that his 'kids are married, they have kids and grandkids and you know. I don't want to become a burden, as far as their lives are concerned.' There seemed to be a hesitancy to rely on others, and a clear desire for more information.

\section{Learning, Changing Behaviour, and Confidence}

The second theme arose from descriptions and perceptions of self-care during the course of the DIVERTCARE intervention. Participants were asked a series of questions for each of the components of the intervention, prompted by an examination of the influence that the intervention component had on daily care management. Most described they learned something new about their medical condition, medications, and managing symptoms. Several elaborated further on the strategies they used to monitor their condition, their increased interest in their own care, and using the zones decision aids.

Karla $(C G, 1)$ articulated her increase in awareness over her condition: 'I would have to say it would open up my eyes an awful lot of different things ... it made me more... attentive and... being more aware and to watch for certain things... which was a real good thing because I had no idea, ya know?' Similarly, Joanne $(C G, 3)$ noticed a change in Keith's $(C, 3)$ attitude towards his health: 'it seems like if you're talking about anything, uh, about his health or anything, like he's right in there, he wants to talk about it, whereas before he was kind of like he would shy away from that conversation type of thing.'

Some respondents described knowledge that they had learned, and were able to teach-back the content as noted by one participant describing inhaler utilization:

I'm pretty well set up. I was to use it twice a day. To keep the little, um, alveoli or whatever call it open; the little sacks in your lung. Keep them open and I don't use it as often now because I don't really... It does help if I have a lot of mucus.'Elizabeth (C, 2)

For some, the information they received was new, and the majority reported gaining knowledge on how their medications worked and strategies to manage symptoms of dyspnea and edema. However, there was one exception where a $\mathrm{HC}$ client had previously participated in education for COPD. He articulated new knowledge regarding the transient nature of chronic diseases and a new awareness of functional impairment. 
'Obviously with emphysema and COPD it's not likely to improve, but it's very nice if you can get it stabilized so it doesn't deteriorate. That's right. I was very well informed. You know, right now I'm down and... you know, it fluctuates a bit around 35\% lung capacity which, you know, as long as they don't over exert myself in high humidity or even at any other... I notice I have limitations, but I know what they are, and I know what to do when I've reached them so.'Mark $(\mathrm{C}, 1)$.

\section{Feeling Connected}

We found an overarching theme woven throughout the participant's interviews, which was a feeling of connection. These findings are presented with three sub-themes: pre-emptive connection, connected to information and medical advice, and connection through the therapeutic relationship.

\section{Preemptive Connection to DIVERT-CARE Professionals}

$\mathrm{HC}$ clients and caregivers in the study reported a feeling of security and connection, which was expressed as continuity and initiation on the part of the care provider. A recurring statement across interviews was appreciation for consistent follow-up regarding health status, whether via an in-home or phone visit.

'It was nice to have her come in and... just to check the heart and lungs, and make sure the medications were okay and so on. We're just grateful that someone was coming in and that someone was available if we needed them!'Paul $(\mathrm{C}, 1)$

The importance of follow-up on timing to diagnosis and intervention was best illustrated in the case of Keith, as told by his caregiver:

'And-and she-she comes in every couple of weeks or so, check it all out and she was floored that his pulse and everything was so low. But I feel like you don't know. What if she didn't come in? It was the perfect time. And God love her. Like if it wasn't for her coming in and doing that, we wouldn't have known that like, you know, he's always had a little bit of issue with his pulse and stuff, but not down in the 30s and that. And with her helping me with that, we tried to figure things out. She got a hold of the doctor about it. They made, um, then the doctor made an appointment with us. He went in, uh, and he ended up getting, uh, a pacemaker put in.' Joanne (CG, 3).

One exception to this theme occurred when additional services were coming into the home. Maureen $(\mathrm{P}, 2)$ expressed that a high frequency of visits led her to book visit-free periods so she could have 'a couple of days to [her]self. The multitude of visits in this case was perceived as a 'nuisance' and not productive.

\section{Connected to Information and Medical Advice}

Over the course of the interviews, many referred to the provided resources, such as the zones decision aid and client education booklets. Participants described scenarios where their health care provider reviewed information and contextualized it to their reality. 
'I got heart failure zones and I've got uh, private community services. I do have that big book that we all got, that health thing that tells you what to do in case of stuff. That big thick one. Remember we got that, who to call and everything, and I look, I refer to that once in a while.'Ellen (C, 2).

However, many reported requiring additional support to digest the information in order to use the information independently. When time was spent reviewing the information and verifying understanding, participants had more confidence in decision-making.

'But I think it was certainly beneficial to have someone that I could discuss my concerns with, such as just giving me an explanation. I know at the time I found it kind of strange that I was on a diuretic and at the same time I was also on a medication to control the frequency of my bladder. I thought, you know, seems to me like you know these two things are working in opposition.' $\operatorname{Jim}(\mathrm{C}, 1)$.

The provision of a medication reconciliation by pharmacists, and reinforced by nursing staff, was also reported to be beneficial. Lori (C, 1) stated, 'I don't think I would've been off as much medication as I am now without all the help I got to do it.'

\section{Connection through Therapeutic Relationships}

The final sub-theme is best described as the emotional connection to the DIVERT-CARE health care provider. Regular visits that occurred over an extended period facilitated therapeutic relationships, and in some cases an emotional bond. Participants expressed that they were satisfied with the program and the health care professionals involved in delivering the intervention components. Words commonly used to describe their interactions included 'comfort', 'caring', and 'understanding'.

'She was good. She was really good, I must say'. Keith $(\mathrm{C}, 3)$

'It was, well I would say a comfort. It was, yeah. It was a [pause]. You know, I think we're blessed to have the care that we get.' Carol (CG, 1).

Participants felt an attachment expressing that they looked forward to the visits and felt comfortable having candid conversations to discuss their cares and concerns. Even participants, like Mark $(C, 1)$, who did not feel like they needed a lot of support expressed that the intervention was mutually beneficial: 'There was nothing I didn't like. It worked alright for me. Anyway, you know, I'm not sorry I went through it, it gave them some insight.' The burden of self-care was apparent, and the gratitude shown by the participants demonstrated the utility and benefits drawn from this intervention. As eloquently summarized by John (CG, 1), 'She treated mom like a person. Not a number'.

\section{Discussion}

We provide a current perspective of chronic disease self-management support from $\mathrm{HC}$ clients regarding symptoms management and the perceived effectiveness of the DIVERT-CARE intervention. Our study highlighted pre-intervention experiences of uncertainty regarding best practices in existing self- 
management of chronic diseases, such as COPD, CHF, and comorbid diabetes. Self-management was also described as burdensome and overwhelming prior to the introduction of the DIVERT-CARE intervention. Our multi-component intervention provided participants with valuable information that was participant-friendly, client-centered, and actionable. As participants reflected on the support they received, a dominant theme of feeling connected illustrates the importance of person-centered approaches. These findings highlight an assessed need for client education and additional support with daily chronic disease management. An assessment of baseline health knowledge is essential in $\mathrm{HC}$ clients, as this information can be used to tailor educational content, knowledge translation strategies and learning needs.

Following participation in the DIVERT-CARE intervention, there was consensus among participants about the utility of health education and client-decision aids. Participants identified the benefit and need for additional health education in the home setting, as it was reported to increase or reinforce health knowledge. However, they also commented on uncertainty about health status and reluctance to act on symptoms associated with disease exacerbations. For example, many struggled in discerning which symptoms (e.g., edema, dyspnea) required the need for medical attention. Participants were hesitant to relay their knowledge gaps and health care needs unless prompted by a healthcare provider. Older adults, similar to our sample, have reported difficulty in decision-making during complex medical situations and express concerns about cognitive capabilities (16). Our findings suggest that pre-determined and consistent support in self-management is a key component that could minimize anxiety about self-care. Additionally, more frequent client engagement increases confidence and is associated with lower health service use (17).

A particular component of health education found to be beneficial was the use of concise and actionable decision aids (e.g., the zones instrument). This tool empowered participants to confidently gauge the severity of their disease state and to act accordingly, which leads to a decrease in miscommunication between providers and clients $(18,19)$. In our study, participants appreciated having 24-hour clinical support available to confer and discuss their understanding of the symptoms they were experiencing and the appropriate clinical interventions moving forward. Based on these findings, we recommend that models of care in the community provide consistent messaging across all educational resources and are congruent with clinician recommendations.

An interesting theme that emerged from this work was the perceived intimacy of therapeutic relationships that developed as a result of having consistent health care providers in the home. Continuity-of-care was an essential component of the DIVERT-CARE trial as it improves client outcomes and allows for the fostering of therapeutic-client relationships (7). Participants appreciated the option of pre-arranged timing of clinical encounters as it provided them with a sense of security and created an opportunity for participatory guidance (20). Continuity-of-care increases client satisfaction and has been shown to decrease health service use $(21,22,23)$. We recommend structuring models of care to promote consistency of caregivers and pre-scheduled wellness check-ups completed in the home. The frequency and intensity of follow-up should be determined in parallel with $\mathrm{HC}$ clients to avoid unnecessary burdens, 
given that one participant was overwhelmed with the number of clinical encounters. Shared-decision making is preferred by clients and may have mitigated this burden pre-emptively (24).

\section{Limitations}

This was a multi-site qualitative study conducted across three provinces. There were several challenges to recruitment and contacting eligible participants. We attempted contact five times spread out over several days, though we were unable to contact a number of clients.

Member checks are typically conducted after thematic analysis to confirm findings. However, we elected to complete informal member checks at the end of the interview to prevent a loss of contact during hospitalizations or due to death following the analysis.

\section{Conclusion}

Participants of a cardio-respiratory management model in $\mathrm{HC}$ reported feelings of uncertainty and a lack of sufficient knowledge to manage care, prior to the intervention. Overall, participants were appreciative and satisfied with the DIVERT-CARE intervention. Health education, comprehensive care in the home, the use of client decision aids, and continuity-of-care were distinct components of the cardio-respiratory management model that participants found beneficial. These factors should be taken into consideration when developing models of care or clinical trials in the community.

\section{Abbreviations}

CHF: Congestive heart failure

COPD: Chronic obstructive pulmonary disease

COREQ: Consolidated Criteria for Reporting Qualitative Research

ED: Emergency departments

HC: Home care

\section{Declarations}

\section{Ethics approval and consent to participate}

This study was approved and conducted according to the policies by the Hamilton Integrated Research Ethics Board (\#4635), Island Health Research Ethics Board (\#C2017-014), and the Newfoundland and Labrador Health Research Ethics Board (\#2018.135). This study was funded by the Canadian Institutes of Health Research (grant \# 148933). 


\section{Consent for publication}

Not applicable

\section{Availability of data and materials}

The datasets used and/or analysed during the current study are available from the corresponding author on reasonable request.

\section{Competing Interests}

The authors declare that they have no competing interests.

\section{Funding}

This study was funded by the Canadian Institutes of Health Research (grant \# 148933). The funding source had no role in the design of the study and data collection, analysis, interpretation of data or writing of the manuscript.

\section{Authors' contributions}

AC is the primary investigator of the DIVERT RCT and conceived the study. CS and DD are experienced in qualitative methods and designed the study. Data was collected, analysed, and interpreted by CS, DD, FM, and LK. The manuscript was prepared by CS, DD, and FM. All authors read, critically revised, and approved the final manuscript.

\section{Acknowledgements}

We would like to thank all the participants for their involvement in the study and to each of the regional home care providers.

\section{References}

1. Costa AP, Schumacher C, Jones A, Dash D, Campbell G, Junek M, et al. DIVERT-Collaboration Action Research and Evaluation (CARE) Trial Protocol: A multiprovincial pragmatic cluster randomised trial of cardiorespiratory management in home care. BMJ Open. 2019; 9: 12.

2. Turcotte M. Canadians with Unmet Homecare Needs. Stat Canada Insights Can Soc 2014;(75-006X):1-12. https://www150.statcan.gc.ca/n1/en/pub/75-006-x/2014001/article/14042-eng.pdf?st=S4cSXo- (1 Dec 2020, date last accessed).

3. Foebel AD, Hirdes JP, Heckman GA, Tyas SL, Tjam EY. A profile of older community-dwelling home care clients with heart failure in Ontario. Chronic Dis Inj Can. 2011;31: 49-57.

4. Nugus P, Holdgate A, Fry M, Forero R, McCarthy S, Braithwaite J. Work pressure and patient flow management in the emergency department: Findings from an ethnographic study. Acad Emerg Med. 
2011; 18: 1045-52.

5. Salvi F, Morichi V, Grilli A, Giorgi R, De Tommaso G, Dessì-Fulgheri P. The elderly in the emergency department: A critical review of problems and solutions. Intern Emerg Med. 2007; 2: 292-301.

6. Kellermann AL, Martinez R. The ER, 50 Years On. N Engl J Med. 2011; 364:2278-9.

7. Schumacher C, Lackey C, Haughton D, Peirce T, Boscart V, Costa AP, et al. A chronic disease management intervention for home care patients with cardio-respiratory symptoms: The DIVERTCARE intervention. Can J Cardiovasc Nurs. 2018; 28 (3): 18-26.

8. Clement C, Edwards SL, Rapport F, Russell IT, Hutchings HA. Exploring qualitative methods reported in registered trials and their yields (EQUITY): Systematic review. BMC Trials. 2018; 19: 1-8.

9. O'Cathain A, Thomas KJ, Drabble SJ, Rudolph A, Hewison J. What can qualitative research do for randomised controlled trials? A systematic mapping review. BMJ Open. 2013; 3: 6.

10. Weiss DJ, Robertson S, Goebel JR. Pilot Implementation of a Low-Literacy Zone Tool for Heart Failure Self-management. J Hosp Palliat Nurs. 2019;21(6):475-81.

11. Clarke V, Braun V. Thematic analysis. J Posit Psychol. 2017; 12: 297-8. http://dx.doi.org/10.1080/17439760.2016.1262613 (1 Dec 2020, date last accessed).

12. Roberts K, Dowell A, Nie JB. Attempting rigour and replicability in thematic analysis of qualitative research data; A case study of codebook development. BMC Med Res Methodol. 2019; 19: 1-8.

13. Morse JM, Barrett M, Mayan M, Olson K, Spiers J. Verification Strategies for Establishing Reliability and Validity in Qualitative Research. Int J Qual Methods. 2002; 1(2): 13-22.

14. Tong A, Sainsbury P, Craig J. Consolidated criteria for reporting qualitative research (COREQ): A 32item checklist for interviews and focus groups. Int J Qual Heal Care. 2007; 19: 349-57.

15. Lincoln YS, Guba EG. Trustworthiness and Naturalistic Evaluation. New Dir Program Eval. 1986; 30.

16. Sun W, Ashtarieh B, Zou P. The safety challenges of therapeutic self-care and informal caregiving in home care: A qualitative descriptive study. Geriatr Nurs (Minneap) . 2020; 000: 1-11. https://doi.org/10.1016/j.gerinurse.2020.07.013 (1 Dec 2020, date last accessed).

17. Barker I, Steventon A, Williamson R, Deeny SR. Self-management capability in patients with long-term conditions is associated with reduced healthcare utilisation across a whole health economy: Crosssectional analysis of electronic health records. BMJ Qual Saf. 2018; 27: 989-99.

18. Liddy C, Blazkho V, Mill K. Challenges of self-management when living with multiple chronic conditions: Systematic review of the qualitative literature. Can Fam Physician. 2014; 60: 1123-33.

19. Östman M, Ung EJ, Falk K. Health-care encounters create both discontinuity and continuity in daily life when living with chronic heart failure - A grounded theory study. Int J Qual Stud Health Wellbeing. 2015; 10: 1-12.

20. Ganann R, Weeres A, Lam A, Chung H, Valaitis R. Optimization of home care nurses in Canada: A scoping review. Heal Soc Care Community. 2019; 27: e604-21.

21. Fan VS, Burman M, McDonell MB, Fihn SD. Continuity of care and other determinants of patient satisfaction with primary care. J Gen Intern Med. 2005; 20: 226-33. 
22. Russell D, Rosati RJ, Rosenfeld P, Marren JM. Continuity in home health care: is consistency in nursing personnel associated with better patient outcomes? J Healthc Qual. 2011; 33(6): 33-9.

23. Klarare A, Rasmussen BH, Fossum B, Fürst CJ, Hansson J, Hagelin CL. Experiences of security and continuity of care: Patients' and families' narratives about the work of specialized palliative home care teams. Palliat Support Care. 2017; 15: 181-9.

24. Chewning B, Bylund CL, Shah B, Arora NK, Gueguen JA, Makoul G. Patient preferences for shared decisions: A systematic review. Patient Educ Couns. 2012; 86: 9-18.

\section{Table}


Table 1

Themes and Supporting Quotes

\section{Theme Additional Quotes}

Self-care

Trajectory

and

Responsibility
Yeah, and like, you know, a lot of people, like a lot of older people, they live on their own and they don't get the proper help that they should because they don't either call or they don't know, you know until it's too late. So and the kids don't, a lot of kids don't care anymore. John (CG, 1)

And so it makes me kind of frail, you know. Alicia $(\mathrm{P}, 3)$

I think I'm [pause] I'm not gunna get better. Because, like I say, but I know, I just hope, I just pray that I don't get worse. Susan $(\mathrm{P}, 1)$

Learning,

Changing

Behaviour, and Confidence

Because I actually listen more and try to understand more and take advice more than I did before. John (CG, 1)

Well, I feel very confident that they know what they're doing and- I don't know, I just feel confident. Maybe that's a result of it. Well, I just feel more aware around when my blood oxygen is low, I don't breathe very well. Alisha $(\mathrm{P}, 2)$

One of the things that she helped me out with is she says "do I weigh myself?" I said "well no" [laughs]. She said, "well that's a very good idea because now that you're on fluid pills or water pills, that'll tell you if everything is working or not because you'll lose weight. If your weight goes up again, it means something is not right or something you're not doing right and you have to get in and get it checked out". So started weighing myself in the morning when I get up and weighing myself in the evenin' before supper. Bob $(\mathrm{P}, 1)$

Well like I said before, once you know the drugs you're taking and you can identify with 'em and you know rough, even if you have no medical background ... they make it clear enough to you which pill is for which - what it's supposed to be doing, then you know which ones for sure you can take or can't forget to take it. Bob $(\mathrm{P}, 1)$

Well, every zone tells you why you're... ya know, obviously if you're in the red zone it's not good at all, but at least if you're in the yellow zone it means to let you know you need to watch something or you need to go back to your doctor or ... like there's a problem coming. Oh, I understood that if you got, if you were silly enough to get back in the yellow zone somewhere you go straight to emerge [/Emergency Department/]. Bob $(\mathrm{P}, 1)$

If you take the medications and follow the instructions they are things that are liveable. Could be worse [laughs]. These are things that can be taken care of in your life, and you can manage 'em and you can go on and live your life as long as you follow the rules and take the pills and whatever. $\operatorname{Bob}(\mathrm{P}, 1)$

She was really good. She got me to understand how to do it properly. I was doing it the wrong way. Mary $(\mathrm{P}, 1)$

Pre-emptive Connection to DIVERT-CARE Professionals
She dealt with and checked out and made sure that I, you know, all my meds - I kept him in a blister pack, and she helped me; showed me some exercises and basically checked out the type of diet that I was on. And yes, I would say initially she came probably over a course of a few days and then she would check up on me primarily by phone. And of course, she was available if I had any problems. $\operatorname{Jim}(\mathrm{P}, 1)$

Just checking up on him was good. Carol (CG, 1) 
She'd be by the next week at a certain date at a certain time and she'd always be there on time. She checked the blood pressure and asked me general questions and how I figure I was doing. Bob $(\mathrm{P}, 1)$

They're going to check in and that also, that was, uh, connecting down to the office. It was hooked up so it would go down to their computer. [home monitoring] Blood pressure run up, they'd phone, or down or if something might be wrong on the readings, they'd phone. Maureen $(\mathrm{P}, 2)$

Connected to Information and Medical Advice
It made it a little more understanding and, you know, things that are happening and that. Karen $(\mathrm{P}, 1)$

Well, she explained everything. Like, you know, we wrote everything all down. She explained about watching the weight. She showed me all the danger zones and what I should watch for. Tammy (CG, 1)

She could tell if we were able to work on our own or if we need that extra assistance because of not being savvy. But you know she did everything: she simplified things, making sure that we were absorbing the information. Donna (CG, 1)

I like to be independent to a certain degree and she allows me that. Doesn't try to tell me "Don't do that because you're not supposed to". It's a form of "let me try something". If I can't do it, I know she's there to back me, to help me. Sarah $(\mathrm{P}, 1)$

Connection through Therapeutic Relationships
And [Nurse] was very patient, very understanding that way. She was a tremendous asset. I have to say, she was really, really awesome... Karla $(\mathrm{CG}, 1)$

For one thing, it's wonderful to talk to somebody else. Um, they'd come in and it's like they're friends, you know? Alisha $(\mathrm{P}, 2)$

\section{Supplementary Files}

This is a list of supplementary files associated with this preprint. Click to download.

- AdditionalFile1 InterviewGuide.docx

- ISSMCOREQChecklistDIVERTCAREExperiencesSupplementFile.pdf 